\title{
De novo variants in CACNA1E found in patients with intellectual disability, developmental regression and social cognition deficit but no seizures
}

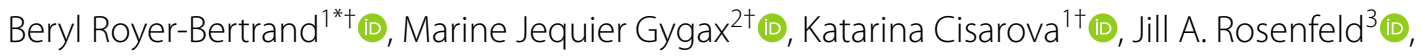

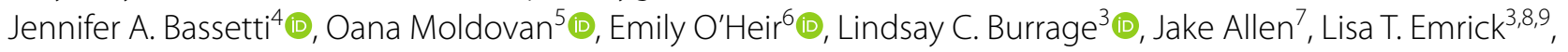
Emma Eastman ${ }^{10}$, Camille Kumps ${ }^{1} \mathbb{B}$, Safdar Abbas ${ }^{11}{ }^{1}$, , Geraldine Van Winckel ${ }^{1}$, Undiagnosed Diseases Network, Nadia Chabane ${ }^{2}$, Elaine H. Zackai ${ }^{12,13}$, Sebastien Lebon ${ }^{14} \mathbb{B}$, Beth Keena ${ }^{12}$, Elizabeth J. Bhoj ${ }^{13,15} \mathbb{B}_{\text {, }}$ Muhammad Umair ${ }^{16,17}$ (D), Dong Li ${ }^{15}$ (D) Kirsten A. Donald ${ }^{18,19}$ (D) and Andrea Superti-Furga ${ }^{1}$ (D)

\begin{abstract}
Background: De novo variants in the voltage-gated calcium channel subunit a1 E gene (CACNA1E) have been described as causative of epileptic encephalopathy with contractures, macrocephaly and dyskinesias.

Methods: Following the observation of an index patient with developmental delay and autism spectrum disorder (ASD) without seizures who had a de novo deleterious CACNA1E variant, we screened GeneMatcher for other individuals with CACNA1E variants and neurodevelopmental phenotypes without epilepsy. The spectrum of pathogenic CACNA1E variants was compared to the mutational landscape of variants in the gnomAD control population database.

Results: We identified seven unrelated individuals with intellectual disability, developmental regression and ASDlike behavioral profile, and notably without epilepsy, who had de novo heterozygous putatively pathogenic variants in CACNA1E. Age of onset of clinical manifestation, presence or absence of regression and degree of severity were variable, and no clear-cut genotype-phenotype association could be recognized. The analysis of disease-associated variants and their comparison to benign variants from the control population allowed for the identification of regions in the CACNA1E protein that seem to be intolerant to substitutions and thus more likely to harbor pathogenic variants. As in a few reported cases with CACNA1E variants and epilepsy, one patient showed a positive clinical behavioral response to topiramate, a specific calcium channel modulator.

Limitations: The significance of our study is limited by the absence of functional experiments of the effect of identified variants, the small sample size and the lack of systematic ASD assessment in all participants. Moreover, topiramate was given to one patient only and for a short period of time.
\end{abstract}

\footnotetext{
*Correspondence: beryl.royer@chuv.ch

†Beryl Royer-Bertrand, Marine Jequier Gygax and Katarina Cisarova contributed equally to this work

${ }^{1}$ Division of Genetic Medicine, Lausanne University Hospital (CHUV) and University of Lausanne, Lausanne, Switzerland

Full list of author information is available at the end of the article Undiagnosed Diseases Network: The list of members is provided as

Supplementary Information.
}

(c) The Author(s) 2021. Open Access This article is licensed under a Creative Commons Attribution 4.0 International License, which permits use, sharing, adaptation, distribution and reproduction in any medium or format, as long as you give appropriate credit to the original author(s) and the source, provide a link to the Creative Commons licence, and indicate if changes were made. The images or other third party material in this article are included in the article's Creative Commons licence, unless indicated otherwise in a credit line to the material. If material is not included in the article's Creative Commons licence and your intended use is not permitted by statutory regulation or exceeds the permitted use, you will need to obtain permission directly from the copyright holder. To view a copy of this licence, visit http://creativecommons.org/licenses/by/4.0/. The Creative Commons Public Domain Dedication waiver (http://creativeco mmons.org/publicdomain/zero/1.0/) applies to the data made available in this article, unless otherwise stated in a credit line to the data. 
Conclusions: Our results indicate that CACNA1E variants may result in neurodevelopmental disorders without epilepsy and expand the mutational and phenotypic spectrum of this gene. CACNA1E deserves to be included in gene panels for non-specific developmental disorders, including ASD, and not limited to patients with seizures, to improve diagnostic recognition and explore the possible efficacy of topiramate.

Keywords: Autism spectrum disorder, CACNA1E, Developmental regression, Epilepsy, Exome sequencing, Global developmental delay, Intellectual disability, Neurodevelopmental disorders, Seizures, Topiramate

\section{Background}

Voltage-gated calcium channels (VGCCs) are proteins that regulate the entry of calcium ions $\left(\mathrm{Ca}^{2+}\right)$ in response to depolarization of the cellular membrane of excitable cells [1] and represent principal gateways for $\mathrm{Ca}^{2+}$ in nerve and both skeletal and cardiac muscle cells [2]. VGCCs are constituted by 5 different subunits, $\alpha 1, \alpha 2, \beta, \delta, \gamma$, which together form the high-voltageactivated $\mathrm{Ca}^{2+}$ channels [3]. The $\alpha 1$ subunit is the main subunit indispensable for the functioning of the channel. It is composed of four homologous domains (I-IV), each containing 6 transmembrane segments, including a voltage sensor segment (S4) and 2 segments forming a pore (S5-S6) [1]. In mammals, ten $\alpha 1$ subunits have been described, encoded by ten different genes. Each of these subunits has specific molecular, genetic, physiologic and pharmacological properties [1, 4], and pathogenic variants in these genes have been associated with various human diseases and represent putative targets for treatment [5].

The $\alpha 1 \mathrm{E}$ gene (CACNA1E) encodes the high-voltage-activated Cav2.3 type $\mathrm{R}$ calcium channel, which is expressed in various areas of the central nervous system, including the cerebellum [1]. More specifically, it is involved in the initiation of the presynaptic calcium entry and post-synaptic transmitter release [6].

CACNA1E variants were initially associated with seizures (Baker JJ 2016: http://epostersonline.com/acmg2 016/node/2302) and with autism spectrum disorder (ASD) [7]. Other studies found a possible impact of CACNA1E single nucleotide polymorphisms in different human diseases and disorders including diabetes [8], migraine [9] and pain regulation [10]. More recently, the pathogenic role of CACNA1E variants in human epilepsy and neurodevelopmental disorders (NDD) has been described. One large genomic study of trios presenting with NDD identified CACNA1E as a candidate gene for NDD with or without epilepsy [11], and subsequently, variants in CACNA1E have been found in patients with developmental and epileptic encephalopathy (DEE) [12]. A large multiplex gene network analysis identified CACNA1E as candidate gene for epilepsy and autism [13]. Subsequently, a genetic association between calcium channels in general and
ASD was further suggested by Liao and $\mathrm{Li}$ [14] and Graziano et al. [15].

The largest cohort of CACNA1E patients, described by Helbig et al. [12], reports 30 patients, aged between 1 and 16 years, who presented with severe to profound developmental delay and a combination of epilepsy, tone disorders and/or abnormal movements. Among these individuals, $88 \%$ were described as non-verbal and nonambulatory. Of the 30 patients, 29 patients presented with hypotonia, six presented with spastic quadriplegia, 13 with joint contractures, 14 with extrapyramidal signs, and 26 with epilepsy (among these, 14 had epileptic spasms). Nine patients presented with developmental regression associated with the onset of epilepsy [12]. Interestingly, topiramate was the main anti-seizure drug reported to show a reduction in seizure presentation. Functional analysis of the Cav2.3 channel in 14 patients revealed a gain-of-function effect of the CACNA1E pathogenic variants with either a facilitated activation or an increased $\mathrm{Ca}^{2+}$ current. As topiramate blocks R-type calcium channels, among its different actions, the R-type calcium current might represent a common neuropathogenic mechanism involved in epilepsy and developmental delay [16].

We identified a de novo missense variant in CACNA1E in a patient with global developmental delay (GDD), ASD and successive developmental regressions unrelated to epileptic seizures. We used GeneMatcher [17] to seek other similar developmental patterns associated with CACNA1E variants, with the aim to identify possible neurodevelopmental presentations without epilepsy and to extend the phenotypic description of CACNA1Erelated disorders. Using large datasets of control populations, we tried to compute the mutational constraint within CACNA1E domains and segments to identify putative functionally relevant segments that are more likely to harbor pathogenic variants.

\section{Method}

\section{Participants}

A missense de novo variant in CACNA1E was found in our index patient after analysis of an extended genetic panel, including epilepsy and developmental delay related genes. His phenotype was notable for two episodes of 
acute regression, in the context of a pre-existing GDD and ASD. Notably, he never presented with any type of epileptic seizures, the common feature in the cohort described by Helbig et al. [12]. Through GeneMatcher, we identified six additional patients who were carriers of heterozygous de novo putative deleterious variants in CACNA1E and presented with GDD, developmental regression and no epilepsy. Except for one patient aged 2, five patients presented with behavioral disturbances belonging to the field of ASD, despite the lack of precise behavioral phenotyping required for an ASD diagnosis. None of these patients has been reported previously.

\section{Sequencing and filtering of variants}

Genomic DNA was extracted from whole blood from the patients and their parents. Exome sequencing in trios was done for $5 / 7$ patients, and in the proband only for $2 / 7$ patients. Segregation of the CACNA1E variants was done by Sanger sequencing for $6 / 7$ patients. The protocols and software versions used for exome capture, sequencing, next-generation sequencing (NGS) pipeline and analysis for each patient are available in Additional file 1: Table S1. All discovered variants are defined in GRCh37 and the NM_000721.4 isoform of CACNA1E, and were classified following guidelines from the American College of Medical Genetics (ACMG) [18].

\section{Analysis of regional intolerance to missense variants in CACNA1E}

We have downloaded the table of Missense Tolerance Ratio (MTR) scores for CACNA1E from MTR-Viewer v2 [19]. MTR is the measure of the proportion of the observed and expected missense variations in a gene given the specific protein regions, reflecting selective constraints of the given region. An MTR score above 1 suggests a positive selection of the segment, MTR score equal to 1 indicates segments' selective neutrality, and an MTR value below 1 indicates purifying selection against missense alterations. A false discovery rate (FDR) value of $<0.1$ is used to determine whether MTR deviates significantly from 1.0, to exclude false positives.

We have calculated the mean MTR score and the mean FDR for each CACNA1E segment, intersegment and interdomain regions. We computed the median, 25th percentile and 5th percentile MTR values $(0.79,0.60$ and 0.33 , respectively). We considered regions being selected against missense variants if their MTR value was below 1 with the associated FDR below 0.1, and being highly intolerant to missense alterations if their MTR value was below the 5th percentile with the respective mean FDR below 0.1 , as previously described [20].

\section{Results}

\section{Clinical reports}

The seven patients, including the index patient and 6 patients recruited via GeneMatcher [17], were all bearing hitherto undescribed de novo heterozygous variants in CACNA1E (see Table 1). Shared clinical features among these patients were GDD, marked speech and language delays and social behavioral deficit. Furthermore, muscular hypotonia, motor stereotypies and sensory issues (a clinical criteria for ASD) were each reported in 5 of 7 patients. Four patients $(4 / 7,57 \%)$ also presented with developmental regression, unrelated to any seizures or specific electro-encephalographic (EEG) epileptic activity. The loss of previously acquired vocabulary and of communicative skills seen in these patients fulfilled the diagnostic criteria for so-called autistic regression. One adult patient (Patient 4) had a history of epileptic spasms, successfully treated with adrenocorticotropic hormone, without subsequent seizures in childhood or adulthood. No recognizable facial phenotype was identified in the patients. None of the patients had epilepsy, spastic quadriplegia, joint contractures, extrapyramidal signs or macrocephaly, as described in Helbig et al. [12]. An overview of the major clinical features seen in our patients is described in Table 1, and a more complete list for each case is available in Additional File 1: Table S2.

The recent articles by Helbig et al. [12] suggested the use of the anti-seizure drug topiramate targeting the Cav2.3 channel, encoded by CACNA1E, in order to manage the refractory epilepsy present in their patients. After identification of a CACNA1E variant in patient 1 in the current study, he received a trial of topiramate on the basis of a hypothesis that even despite the absence of seizures, modulation of intracellular $\mathrm{Ca}^{2+}$ influx might positively impact the behavioral phenotype. For this reason, we present a more detailed clinical history of this patient.

Patient 1 presented with marked developmental regression at age of 14 months with loss of first words and communicative gestures. Retrospectively, the development up to that point had been slow, particularly concerning speech development and communication competencies. Subsequently, there was slow progress in language acquisition, and at age 4 years, he was diagnosed with ASD and GDD. At that time, the first regression episode was interpreted as suggestive of an autistic regression.

At age 5 years, he suddenly presented with a second developmental regression with motor hyperactivity, pervasive stereotypies (body rocking and hand stereotypies), loss of interest in play, and loss of sphincter control at night. Brain MRI and a metabolic assessment were normal. EEG showed bilateral occipital spikes, activated during sleep. He never had epileptic seizures. He had a severe sleep disorder. The sleep EEG monitoring 


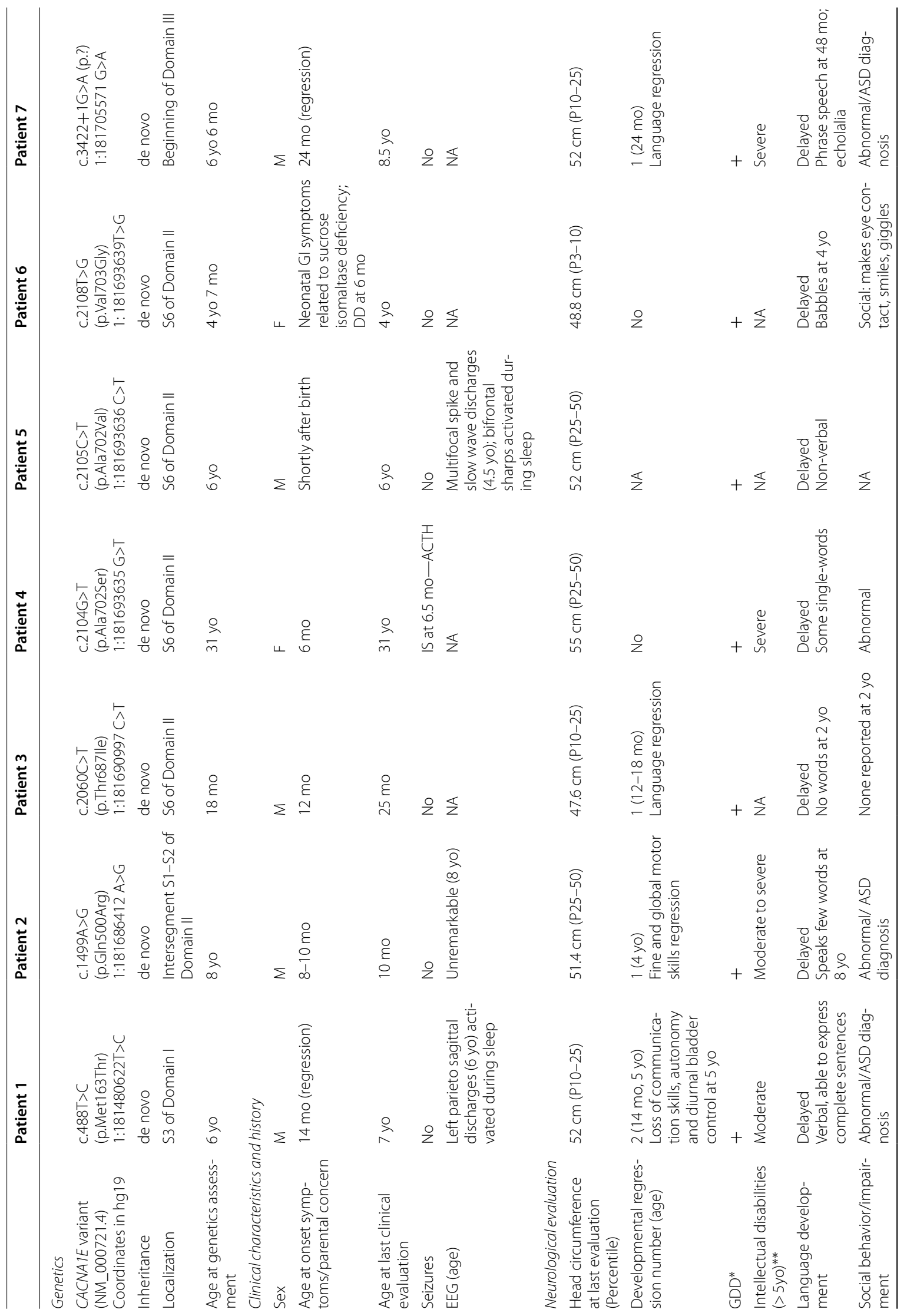




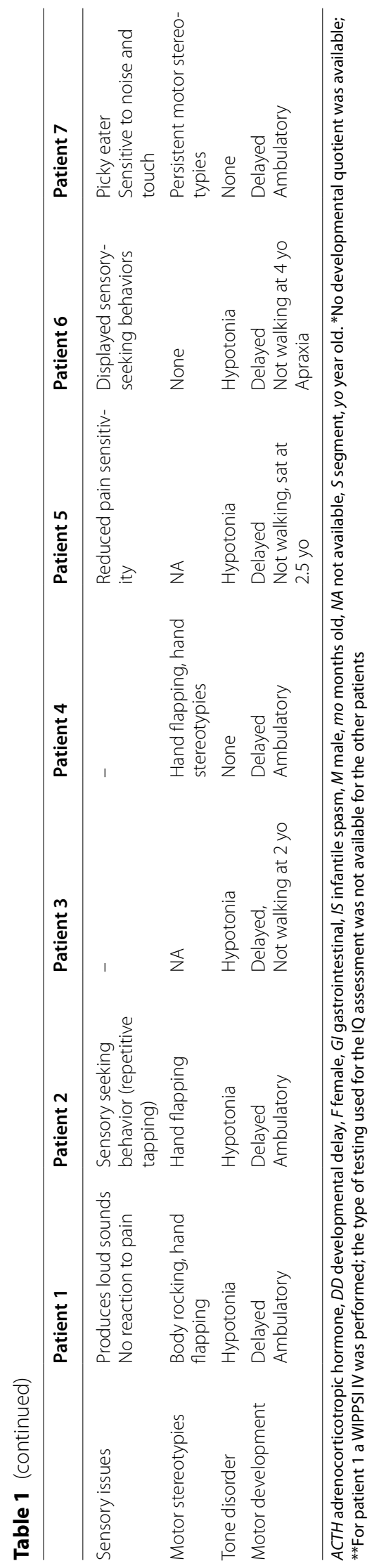


excluded nocturnal epileptic seizures as the cause of multiple awakenings. Progressive titration of topiramate up to $2 \mathrm{mg} / \mathrm{kg} /$ day was accompanied by improvement in motor hyperkinesia and social contact, and regain of nocturnal sphincter control. A dosage above $2.5 \mathrm{mg} / \mathrm{kg} / \mathrm{day}$ was not well tolerated because of increased irritability.

\section{Genetics findings}

With exome sequencing, we identified seven novel variants in CACNA1E in seven unrelated patients. In individual 1 , a de novo missense mutation in CACNA1E, c. $488 \mathrm{~T}>\mathrm{C}$, was localized in the segment S3 of Domain I, leading to a p.Met163Thr change. Individual 2 carried a c. $1499 \mathrm{~A}>\mathrm{G}$ de novo missense variant in the Domain II of CACNA1E, between segments S1 and S2, leading to a p.Gln500Arg change. Individuals 3 to 6 carried each a de novo missense variant clustering in the segment $\mathrm{S} 6$ of the Domain II, c.2060C $>\mathrm{T}$, c.2104G $>\mathrm{T}$, c.2105C $>\mathrm{T}$ and c.2108T $>\mathrm{G}$, resulting in p.Thr687Ile, p.Ala702Ser, p.Ala702Val and p.Val703Gly changes, respectively. Individual 7 carried a de novo splicing variant c. $3422+1 \mathrm{G}>\mathrm{A}$, localized at the beginning of Domain III.

These variants' localizations on the CACNA1E structure and domains are shown in Fig. $1 \mathrm{~A}$, along with the variants described in Helbig et al. [12] and in Heyne et al. [11]. All residues impacted by these missense variants in individuals 1-6 are highly conserved and predicted to be deleterious by various in silico predictors (Additional file 1: Table S3). The splicing variant in individual 7 is predicted by three splicing predictors (MaxEntScan, NNSPLICE, and Human Splicing Finder from Alamut Visual (Interactive Biosoftware)) to disrupt exon 22 consensus donor site. None of the seven novel variants reported here are present in public databases of control individuals (gnomAD [21], LOVD [22]).

CACNA1E is predicted to be associated mainly with an autosomal dominant disorder, with a high DOMINO score of 0.995 [23]. Bioinformatic analysis of variant types and distributions in gnomAD control dataset revealed a conspicuous absence of loss-of-function (LOF) variants in CACNA1E, suggesting that this gene is highly intolerant to truncating mutations (gnomAD LOF metrics for CACNA1E: $\mathrm{pLI}=1$ and $\mathrm{LOEUF}=0.12$ ). CACNA1E is also strongly intolerant to missense variation (gnomAD $\mathrm{Z}$ score $=5.81)$ [21].

We used MTR region-gated analysis of missense variants [19] to infer the functional importance of the different segments and domains of the protein (Fig. 2). The significantly conserved regions (corresponding to MTR score below 0.33) are located in the segment 6 of Domains I and II as well as the regions just at the end of Domains I and III and the intersegment S4-S5 in Domain I (Fig. 2). It correlates with the different clusters of pathogenic variants observed in the reported patients (Fig. 1A).

\section{Discussion}

The seven individuals (six children and one young adult) with heterozygous de novo pathogenic variants in $C A C$ $N A 1 E$ described here showed a broad phenotypic spectrum including intellectual disability, GDD, abnormal behavioral phenotypes (in three cases associated with clinically confirmed ASD diagnosis), hypotonia, absence of language or speech delay and developmental regressions in infancy, but no severe neuromotor deficit or epileptic seizures (except for epileptic spasms in Patient 4). This cohort has enabled us to broaden the genotypic and the phenotypic spectrum of $C A C N A 1 E$-related neurodevelopmental disorder.

The phenotype in our patients was milder than the one reported previously [12], which might be linked to the absence of refractory epilepsy. Indeed, the 30 patients previously reported [12] presented with a more severe neurodevelopmental phenotype, as the expression of a more severely impacted brain developmental trajectory. Among them, four had no epilepsy and one had no information available for the epilepsy phenotype. The nonepileptic patients older than 2 years $(2 / 4,50 \%)$ showed a milder phenotype than the others in the cohort, having single-word capacity and being ambulant. Cognitive and behavioral profiles reported for those patients were very similar to the ones seen in our cohort. However, social deficits were not reported in their study.

Pathogenic CACNA1E variants reported previously [12] and in this paper cluster mostly inside or at the end of segment 6 of Domains I, II and III (Fig. 1A). Based on the analysis of genetic intolerance, those regions are the most intolerant to missense variation (Fig. 2). Thus, the natural variant epidemiology supports the concept that these regions are most crucial for the normal function of CACNA1E [16, 24].

Based on the clinical description of the patients harboring the different CACNA1E variants, there seems to be no clear link between the localization of the variant on the protein and the clinical outcome, especially of refractory epilepsy or spastic quadriplegia (Fig. 1B). Indeed, four of the patients presented here carry missense variants impacting either the same amino acid (p.Ala702) or in the same clustered region of the segment 6 in Domain II as some patients reported with severe epilepsy [12]. Interestingly, among the patients without epilepsy reported previously, two harbor missense variants also seen in patients with severe epilepsy [12]. The first patient is a 1-year-old girl with a p.Gly352Arg missense variant, a recurrent mutation seen in 8 other patients with a strong epilepsy phenotype, which started after 


\begin{tabular}{l} 
Enilepsy \\
\hline GDD
\end{tabular}

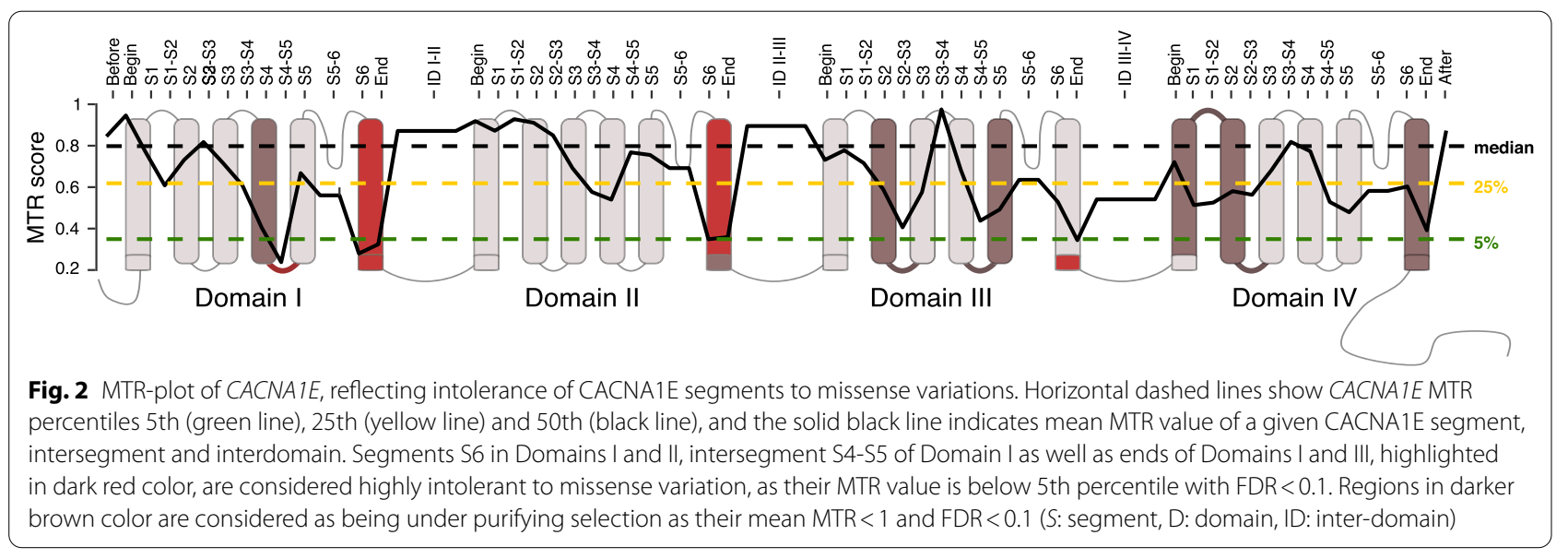

age 1 in 3/8 cases. The second non-epileptic patient is a 6-year-old boy carrying a p.Thr1425Asn variant, also seen in a second patient who had epileptic spasms at 5 months of age, but none since then.
Based on the existing reports as well as the case series reported here, pathogenic variants in CACNA1E appear to result in a disease with a spectrum of clinical manifestations ranging from severe refractory epilepsy 
with globally, severely disturbed development (DEE) to GDD with intellectual disability, developmental regression and ASD. This high phenotypic heterogeneity is not uncommon in both adult and developmental channelopathies [25], and it is made even more evident by the observation of different phenotypes resulting from identical mutations. Indeed, it is likely that other mechanisms including modifier genes, environmental factors or variations in gene expressivity contribute to the variability of the phenotypes. Considering the variability in the age of epileptic onset in the cohort of Helbig et al. [12], ranging from 1 day to 3 years, we cannot exclude that the younger patients in our cohort may develop seizures later in life.

The identification of a splicing variant adds further complexity to the pathogenic mechanisms. Our patient 7 has a variant predicted to disrupt exon 22 consensus splicing donor site $(\mathrm{c} .3422+1 \mathrm{G}>\mathrm{A})$. Helbig et al, have tested electrophysiologically the functional effects of the three CACNA1E missense variants in the segment 6 of Domain II and one variant located in the S4-S5 intersegment of Domain II. These 4 missense variants all led to an increase in current density, facilitated channel activation and slower deactivation, and were thus consistent with gain-of-function effect of the variants. Additionally, the study also described three loss-of-function variants impacting CACNA1E and suggested that haploinsufficiency might be another pathogenic mechanism. The phenotypes of those three patients were milder than the others in their study and resemble that of the patients in our series. The presence of both haploinsufficiency variants as well as missense variants that may act through dominant negative or perhaps even gain-of-function mechanism is intriguing, but not novel. The existence of both loss-of-function and gain-of-function variants in calcium channel genes has been reported in CACNA1A for variants leading to variable severity of DEE [26], as well as in other neuronal channels (SCN8A [27], KCNQ2 [28], KCNQ5 [29], KCNA2 [30], KCNB1 [31]). Interestingly, gain-of-function changes in the electrophysiology of the cell can also result from loss-of-function effects of variants at the molecular level, and therefore, the gainof-function effect of truncating and/or splicing variants in CACNA1E should not be excluded without further functional studies of these variants. Based on the genetic epidemiology of variants in gnomAD and our computations, the CACNA1E gene seems to be constrained both against missense as well as against loss-of-function variants. Thus, the pathogenic mechanism remains unclear and this complicates genotype-phenotype correlation analysis. Such molecular understanding would be therefore needed in the future in order to design therapeutic approaches with drugs such as topiramate.
Furthermore, the observations in this small cohort challenge our current understanding of the interplay between epilepsy and its impact versus its co-occurrence with developmental delay. The term DEE was coined by the most recent classification from the International League Against Epilepsy (ILAE), defining relationship between developmental delay with or without early-life pharmacoresistant epilepsy [32]. However, recent publications of subjects harboring pathogenic variants in socalled epilepsy genes who did not have seizures suggest that the concept of variants specific to epilepsy in the context of syndromic presentation may be mistaken. In that aspect, epilepsy, or ASD, might be considered as one of the phenotypic expressions of a monogenic syndrome [33]. The present work adds CACNA1E to the expanding list of genes implicated in an autosomal dominant neurodevelopmental disorder, including ASD, with or without epilepsy. Growing evidence suggests that diagnostic gene panels for epilepsy should be merged with larger brain development gene panels.

In Patient 1, treatment of topiramate [12] was introduced with the hypothesis that modulation of the dysfunctional $\alpha 1$ subunit might improve the function of the neuronal connectivity underlying behavioral disorders, independently of the electroencephalographic activity as the patient never presented with any seizure. Parents reported a qualitative improvement concerning sleep quality and sphincter control; however, it might had happened by chance or as a natural evolution of the disorder. No additional effect has been observed on his behavioral disorder and development.

This study provides a further example of how matchmaking tools like GeneMatcher can be used to define expanded phenotypes associated with described disease genes, like it has been previously described for $L M N B 1$ [34] and GATAD2B [35]. As NGS analysis is becoming routine worldwide and many laboratories are faced with variants that may not correspond to "typical" phenotypes, these sharing and matchmaking tools will become even more important to facilitate the recognition of novel phenotypic associations and variant interpretation.

\section{Limitations}

The main limitation of our study is the absence of functional studies of the effects and impacts of the identified variants on Cav2.3 channel function. Due to this lack of experimental data, we cannot assess whether the variants reported have a gain-of-function effect, as described in Helbig et al. [12]. Considering the similar types of variants (missense) and the consistent NDD phenotype, the de novo origin of these variants, their absence in control population, their impact on conserved amino acid and deleterious predictions and the 
presence of nearby pathogenic variants, our reported variants are classified as likely pathogenic/pathogenic according to ACMG criteria [18]. Functional experiments are nevertheless essential to understand the pathomechanism behind the reported variants.

The second limitation of our study is the small cohort size. Gathering of patients through matchmaking platforms comes with clear benefits but at the same time is limited by its multi-centric nature, which leads to involvement of variable specialists and assessment tools for each patient. Similarly, the individual submitting the information on the matchmaking platforms might not always be in direct contact with the patients and their referring clinicians, explaining the variability in clinical assessments and detailed history of the patients, for example here the presence/absence of early regression or diagnostic criteria and full assessment for ASD. It thus shows how crucial the collaboration between clinicians and molecular specialists might be in the future.

Topiramate was tested in only one patient of the cohort, and no specific recommendations can be made, as the indication for its use was speculative. However, this information might support the use of this anti-seizure medication drug in unusual indications.

In addition, our study highlights the role of both splicing loss-of-function and missense variants but does not elucidate the underlying molecular mechanisms.

\section{Conclusion}

In this study, we identified heterozygous pathogenic or likely pathogenic de novo variants impacting $C A C$ $N A 1 E$ in seven patients presented with a phenotype that was less severe than that previously reported, notably with the absence of severe epilepsy, of macrocephaly and of spastic quadriplegia. The overlapping phenotypes in our patients include GDD, abnormal social behavior including ASD, hypotonia and developmental regression. Furthermore, we analyzed the variant landscape of CACNA1E and showed strong constraint of missense variants in specific segments of CACNA1E. We conclude that $C A C N A 1 E$ variants have a broader phenotypic spectrum than previously reported, including non-epileptic ASD and GDD, and this may inform the composition of diagnostic gene panels and the interpretation of variants observed in NGS studies.

\section{Abbreviations}

ACMG: The American College of Medical Genetics; ASD: Autism spectrum disorder; DEE: Developmental and/or epileptic encephalopathy; FDR: False Discovery Rate; GDD: Global developmental delay; MTR: Missense Tolerance Ratio; NDD: Neurodevelopmental disorder; NGS: Next-Generation Sequencing; VGCC: Voltage-gated calcium channels.

\section{Supplementary Information}

The online version contains supplementary material available at https://doi. org/10.1186/s13229-021-00473-3.

Additional file 1: Table S1. Sequencing methods for each patient of the cohort. Table S2. Detailed clinical description of CACNA1E patients. Table 3. Variants' description, bioinformatics predictors and ACMG classification.

Additional file 2. List of members of the Undiagnosed Diseases Network.

\section{Acknowledgements}

The authors are grateful to the patients and to their families for their participation in this study.

\section{Authors' contributions}

BRB, MJG, KC contributed to conceptualization. BRB, MJG, KC, ASF prepared the original draft. All the authors contributed to writing, reviewing and editing. $B R B, D L, O M, J A B, M U$ are the persons of contact on GeneMatcher. BRB, $K C, L C B, D L, E J B$ contributed to analysis. MJG, JAR, BK, OM, JAB, SL, GVW, MU contributed to clinical evaluation. MJG, LTM, EHZ, OM, JAB, SL, CK, GVW, NC are the physicians following the patients. All authors read and approved the final manuscript.

\section{Funding}

BRB received the support of the "Jeune Chercheur" grant 2020 of the Department of Medicine of the Lausanne University Hospital - CHUV. Sequencing and analysis of Patient 6 were provided by the Broad Institute of MIT and Harvard Center for Mendelian Genomics (Broad CMG) and were funded by the National Human Genome Research Institute, the National Eye Institute, and the National Heart, Lung and Blood Institute grant UM1 HG008900 and in part by National Human Genome Research Institute grant R01 HG009141. NeuroDev is supported by the Stanley Centre for Psychiatric Research at the Broad Institute and the National Institute of Mental Health. This work was supported by a grant from the Simons Foundation/SFARI (599648, E.R., A.A., C.R.N, K.A.D). Research reported in this manuscript was supported by the NIH Common Fund, through the Office of Strategic Coordination/Office of the NIH Director under Award Number U01HG007709. The content is solely the responsibility of the authors and does not necessarily represent the official views of the National Institutes of Health.

\section{Declarations}

\section{Ethics approval and consent to participate}

All subjects provided written informed consent, obtained from their legal guardians, and the study was conducted in adherence with the Declaration of Helsinki.

\section{Consent for publication}

All subjects consented for publication.

\section{Competing interests}

The Department of Molecular and Human Genetics at Baylor College of Medicine receives revenue from clinical genetic testing completed at Baylor Genetics Laboratories.

\section{Availability of data and materials}

The genetic data supporting the findings of this study, as a whole, contain information that could compromise the privacy and/or consent of the participants. We therefore only provide the causative variants' details, which are available in the manuscript and tables.

\footnotetext{
Author details

${ }^{1}$ Division of Genetic Medicine, Lausanne University Hospital (CHUV) and University of Lausanne, Lausanne, Switzerland. ${ }^{2}$ Division of Autistic Spectrum Disorders, Department of Psychiatry, Lausanne University Hospital (CHUV), Lausanne, Switzerland. ${ }^{3}$ Department of Molecular and Human Genetics, Baylor College of Medicine, Houston, TX, USA. ${ }^{4}$ Division of Medical Genetics,
} 
Department of Pediatrics, Weill Cornell Medicine, New York, NY, USA. ${ }^{5}$ Serviço de Genética Médica, Departamento de Pediatria, Hospital de Santa Maria, Centro Hospitalar Universitário de Lisboa Norte, Lisbon, Portugal. ${ }^{6}$ Center for Mendelian Genomics and Program in Medical and Population Genetics, Broad Institute of MIT and Harvard, Cambridge, MA, USA. ${ }^{7}$ The Broad Institute of MIT and Harvard, Cambridge, MA, USA. ${ }^{8}$ Department of Neurology, Baylor College of Medicine, Houston, TX, USA. ${ }^{9}$ Department of Pediatrics, Baylor College of Medicine, Houston, TX, USA. ${ }^{10}$ Department of Paediatrics and Child Health, Faculty of Health Sciences, University of Cape Town, Cape Town, South Africa. ${ }^{11}$ Department of Biochemistry, Faculty of Biological Sciences, Quaid-i-Azam University, Islamabad, Pakistan. ${ }^{12}$ Division of Human Genetics, Department of Pediatrics, The Children's Hospital of Philadelphia, Philadelphia, PA, USA. ${ }^{13}$ Department of Pediatrics, Perelman School of Medicine at the University of Pennsylvania, Philadelphia, PA, USA. ${ }^{14}$ Unit of Paediatric Neurology and Pediatric Neurorehabiliation, Woman-Mother-Child Department, Lausanne University Hospital, Lausanne, Switzerland. ${ }^{15}$ Center for Applied Genomics, The Children's Hospital of Philadelphia, Philadelphia, PA, USA. ${ }^{16}$ Medical Genomics Research Department, King Abdullah International Medical Research Center (KAIMRC), King Saud Bin Abdulaziz University for Health Sciences, Ministry of National Guard Health Affairs, Riyadh, Saudi Arabia. ${ }^{17}$ Department of Life Sciences, School of Science, University of Management and Technology (UMT), Lahore, Pakistan. ${ }^{18}$ Department of Paediatrics and Child Health, Red Cross War Memorial Children's Hospital, Cape Town, South Africa. ${ }^{19}$ Neuroscience Institute, Faculty of Health Sciences, University of Cape Town, Cape Town, South Africa.

Received: 28 June 2021 Accepted: 1 October 2021 Published online: 26 October 2021

\section{References}

1. Catterall WA. Voltage-gated calcium channels. Cold Spring Harb Perspect Biol. 2011;3(8):a003947.

2. Buraei Z, Lumen E, Kaur S, Yang J. RGK regulation of voltage-gated calcium channels. Sci China Life Sci. 2015;58(1):28-38.

3. Tanabe T, Takeshima H, Mikami A, Flockerzi V, Takahashi H, Kangawa K, et al. Primary structure of the receptor for calcium channel blockers from skeletal muscle. Nature. 1987;328(6128):313-8.

4. Lipscombe D, Allen SE, Toro CP. Control of neuronal voltagegated calcium ion channels from RNA to protein. Trends Neurosci. 2013;36(10):598-609.

5. Phan NN, Wang CY, Chen CF, Sun ZD, Lai MD, Lin YC. Voltage-gated calcium channels: novel targets for cancer therapy. Oncol Lett. 2017;14(2):2059-74.

6. Parajuli LK, Nakajima C, Kulik A, Matsui K, Schneider T, Shigemoto $R$, et al. Quantitative regional and ultrastructural localization of the $\mathrm{Ca}(\mathrm{v}) 2.3$ subunit of R-type calcium channel in mouse brain. J Neurosci. 2012;32(39):13555-67.

7. Breitenkamp AF, Matthes J, Herzig S. Voltage-gated calcium channels and autism spectrum disorders. Curr Mol Pharmacol. 2015;8(2):123-32.

8. Trombetta M, Bonetti S, Boselli M, Turrini F, Malerba G, Trabetti E, et al. CACNA1E variants affect beta cell function in patients with newly diagnosed type 2 diabetes. The Verona newly diagnosed type 2 diabetes study (VNDS) 3. PLoS ONE. 2012;7(3):e32755.

9. Ambrosini A, D'Onofrio M, Buzzi MG, Arisi I, Grieco GS, Pierelli F, et al. Possible involvement of the CACNA1E gene in migraine: a search for single nucleotide polymorphism in different clinical phenotypes. Headache. 2017:57(7):1136-44.

10. Amano K, Nishizawa D, Mieda T, Tsujita M, Kitamura A, Hasegawa J, et al. Opposite associations between the rs3845446 single-nucleotide polymorphism of the CACNA1E gene and postoperative pain-related phenotypes in gastrointestinal surgery versus previously reported orthognathic surgery. J Pain. 2016;17(10):1126-34.

11. Heyne HO, Singh T, Stamberger $H$, Abou Jamra R, Caglayan $H$, Craiu D, et al. De novo variants in neurodevelopmental disorders with epilepsy. Nat Genet. 2018;50(7):1048-53.

12. Helbig KL, Lauerer RJ, Bahr JC, Souza IA, Myers CT, Uysal B, et al. De novo pathogenic variants in CACNA1E cause developmental and epileptic encephalopathy with contractures, macrocephaly, and dyskinesias. Am J Hum Genet. 2018;103(5):666-78.
13. Peng J, Zhou Y, Wang K. Multiplex gene and phenotype network to characterize shared genetic pathways of epilepsy and autism. Sci Rep. 2021;11(1):952.

14. Liao X, Li Y. Genetic associations between voltage-gated calcium channels and autism spectrum disorder: a systematic review. Mol Brain. 2020;13(1):96.

15. Graziano C, Despang P, Palombo F, Severi G, Posar A, Cassio A, et al. A new homozygous CACNB2 mutation has functional relevance and supports a role for calcium channels in autism spectrum disorder. J Autism Dev Disord. 2021;51(1):377-81.

16. Carvill GL. Calcium channel dysfunction in epilepsy: gain of CACNA1E. Epilepsy Curr. 2019;19(3):199-201.

17. Sobreira N, Schiettecatte F, Valle D, Hamosh A. GeneMatcher: a matching tool for connecting investigators with an interest in the same gene. Hum Mutat. 2015;36(10):928-30.

18. Richards S, Aziz N, Bale S, Bick D, Das S, Gastier-Foster J, et al. Standards and guidelines for the interpretation of sequence variants: a joint consensus recommendation of the American College of Medical Genetics and Genomics and the Association for Molecular Pathology. Genet Med. 2015;17(5):405-24.

19. Traynelis J, Silk M, Wang Q, Berkovic SF, Liu L, Ascher DB, et al. Optimizing genomic medicine in epilepsy through a gene-customized approach to missense variant interpretation. Genome Res. 2017;27(10):1715-29.

20. Li GC, Forster-Benson ETC, Sanders CR. Genetic intolerance analysis as a tool for protein science. Biochim Biophys Acta Biomembr. 2020;1862(1):183058.

21. Karczewski KJ, Francioli LC, Tiao G, Cummings BB, Alfoldi J, Wang Q, et al. Author correction: The mutational constraint spectrum quantified from variation in 141,456 humans. Nature. 2021:590(7846):E53.

22. Fokkema IF, Taschner PE, Schaafsma GC, Celli J, Laros JF, den Dunnen JT. LOVD v.2.0: the next generation in gene variant databases. Hum Mutat. 2011;32(5):557-63.

23. Quinodoz M, Royer-Bertrand B, Cisarova K, Di Gioia SA, Superti-Furga A, Rivolta C. DOMINO: using machine learning to predict genes associated with dominant disorders. Am J Hum Genet. 2017;101(4):623-9.

24. Xie C, Zhen XG, Yang J. Localization of the activation gate of a voltagegated Ca2+ channel. J Gen Physiol. 2005;126(3):205-12.

25. Smith RS, Walsh CA. Ion channel functions in early brain development. Trends Neurosci. 2020;43(2):103-14.

26. Jiang X, Raju PK, D'Avanzo N, Lachance M, Pepin J, Dubeau F, et al. Both gain-of-function and loss-of-function de novo CACNA1A mutations cause severe developmental epileptic encephalopathies in the spectrum of Lennox-Gastaut syndrome. Epilepsia. 2019;60(9):1881-94.

27. Blanchard MG, Willemsen MH, Walker JB, Dib-Hajj SD, Waxman SG, Jongmans MC, et al. De novo gain-of-function and loss-of-function mutations of SCN8A in patients with intellectual disabilities and epilepsy. J Med Genet. 2015;52(5):330-7.

28. Miceli F, Soldovieri MV, Ambrosino P, De Maria M, Migliore M, Migliore R, et al. Early-onset epileptic encephalopathy caused by gain-of-function mutations in the voltage sensor of Kv7.2 and Kv7.3 potassium channel subunits. J Neurosci. 2015;35(9):3782-93.

29. Lehman A, Thouta S, Mancini GMS, Naidu S, van Slegtenhorst M, McWalter K, et al. Loss-of-function and gain-of-function mutations in KCNQ5 cause intellectual disability or epileptic encephalopathy. Am J Hum Genet. 2017;101(1):65-74.

30. Syrbe S, Hedrich UBS, Riesch E, Djemie T, Muller S, Moller RS, et al. De novo loss- or gain-of-function mutations in KCNA2 cause epileptic encephalopathy. Nat Genet. 2015;47(4):393-9.

31. Torkamani A, Bersell K, Jorge BS, Bjork RL Jr, Friedman JR, Bloss CS, et al. De novo KCNB1 mutations in epileptic encephalopathy. Ann Neurol. 2014;76(4):529-40.

32. Fisher RS, Cross JH, French JA, Higurashi N, Hirsch E, Jansen FE, et al. Operational classification of seizure types by the International League Against Epilepsy: Position Paper of the ILAE Commission for Classification and Terminology. Epilepsia. 2017;58(4):522-30.

33. Specchio N, Curatolo P. Developmental and epileptic encephalopathies: what we do and do not know. Brain. 2021;144(1):32-43.

34. Cristofoli F, Moss T, Moore HW, Devriendt K, Flanagan-Steet H, May M, et al. De Novo variants in LMNB1 cause pronounced syndromic microcephaly and disruption of nuclear envelope integrity. Am J Hum Genet. 2020;107(4):753-62. 
35. Vera G, Sorlin A, Delplancq G, Lecoquierre F, Brasseur-Daudruy M, Petit F, et al. Clinical and molecular description of 19 patients with GATAD2BAssociated Neurodevelopmental Disorder (GAND). Eur J Med Genet. 2020;63(10):104004

\section{Publisher's Note}

Springer Nature remains neutral with regard to jurisdictional claims in published maps and institutional affiliations.
Ready to submit your research? Choose BMC and benefit from:

- fast, convenient online submission

- thorough peer review by experienced researchers in your field

- rapid publication on acceptance

- support for research data, including large and complex data types

- gold Open Access which fosters wider collaboration and increased citations

- maximum visibility for your research: over $100 \mathrm{M}$ website views per year

At BMC, research is always in progress.

Learn more biomedcentral.com/submissions 